Recibido: 08/06/2020 --- Aceptado: 21/08/2020 --- Publicado: 15/11/2020

\title{
ESTUDIO COMPARATIVO SOBRE LAS EXPECTATIVAS LABORALES DE ESTUDIANTES DE LA CARRERA DE EDUCACIÓN INICIAL Y DE ESTUDIANTES DE PROFESIONALIZACIÓN EN SERVICIO EN LA UNIVERSIDAD METROPOLITANA.
}

\section{COMPARATIVE STUDY ABOUT LABOR EXPECTATIONS OF STUDENTS OF THE INITIAL EDUCATION CAREER AND PROFESSIONALIZATION AT THE METROPOLITAN UNIVERSITY}

Natalia Castañón Octavio¹. Universidad Metropolitana. Venezuela. castanon@unimet.edu.ve

Giovanna González Reyes. Universidad Metropolitana. Venezuela. giovannagonzalez@correo.unimet.edu.ve

Valeria Lupo Carbone. Universidad Metropolitana. Venezuela.

valeria.lupo@correo.unimet.edu.ve

\section{RESUMEN}

El presente estudio tiene como objetivo principal comparar las expectativas laborales de los estudiantes de la carrera de Educación Inicial con las de los estudiantes del Programa de Profesionalización en Servicio de la Universidad Metropolitana, aunado a esto posee tres objetivos específicos que constan de indagar y constatar las expectativas laborales de ambos grupos y describir ambos perfiles de ingreso. La presente investigación no experimental de tipo descriptivo y comparativo con metodología cuantitativa logró determinar que existen múltiples semejanzas y diferencias entre ambos grupos de estudio destacando la principal: los estudiantes de profesionalización entran en la carrera con experiencia laboral, mientras que los estudiantes de educación inicial entran en la carrera con poca o nula experiencia laboral. Al igual que las principales investigaciones de las que bebe esta, su fundamento está basado en la idea de comunicación sostenible, entendida como transparencia en la previsión de expectativas laborales de un sector fundamental para la consecución de desarrollo humano sostenible.

\footnotetext{
${ }^{1}$ Natalia Castañón Octavio: Profesora e investigadora en la Universidad Metropolitana. Licenciada en Educación (Universidad Metropolitana, 1991). Maestría en Psicología Escolar (Universidad Complutense de Madrid, 1993). Doctorada en Filosofía y Ciencias de la Educación (Universidad Complutense de Madrid, 1999)
} 
Castañón Octavio, N., González Reyes, G., y Lupo Carbone, V. Estudio comparativo sobre las expectativas laborales de estudiantes de la carrera de educación inicial y los estudiantes de profesionalización en servicio en la Universidad Metropolitana

PALABRAS CLAVE: expectativas laborales - profesionalización - estudiantes universitarios - trabajo - educación.

\section{ABSTRACT}

The main objective of this study is to compare the labor expectations of the students of the Initial Education degree with those of the students of the Service Professionalization Program of the Metropolitan University, in addition to this, it has three specific objectives that consist of investigating and verifying the job expectations of both groups and describe both income profiles. The present nonexperimental descriptive and comparative research with quantitative methodology was able to determine that there are multiple similarities and differences between the two study groups, highlighting the main one: professionalization students enter the career with work experience, while initial education students enter in the career with little or no work experience. Like the main papers from which ours drinks, its foundations are based on the idea of sustainable communication, understood as transparency in the forecast of labor expectations of a fundamental sector in the achievement of sustainable human development.

KEY WORDS: labor expectations - professionalization - university students - job education.

\section{ESTUDO COMPARATIVO DAS EXPECTATIVAS TRABALHISTAS DE ESTUDANTES DA CARREIRA DE EDUCAÇÃO INICIAL E DE ESTUDANTES DE PROFISSIONALIZAÇÃO EM SERVIÇO NA UNIVERSIDADE METROPOLITANA}

\section{RESUMO}

O principal objetivo deste estudo é comparar as expectativas de trabalho dos alunos do curso de Educação Inicial com as dos alunos do Programa de Profissionalização de Serviços da Universidade Metropolitana, além disso, possui três objetivos específicos que consistem em investigar e verificar a expectativas de trabalho de ambos os grupos e descrever os dois perfis de renda. A presente pesquisa descritiva e comparativa não experimental, com metodologia quantitativa, foi capaz de determinar que existem múltiplas semelhanças e diferenças entre os dois grupos de estudo, destacando o principal: estudantes de profissionalização iniciam a carreira com experiência de trabalho, enquanto estudantes de educação inicial entram na carreira com pouca ou nenhuma experiência de trabalho.

Como a principal pesquisa de que bebe, sua base é baseada na ideia de comunicação sustentável, entendida como transparência na previsão das expectativas de trabalho de um setor fundamental para alcançar o desenvolvimento humano sustentável.

PALAVRAS CHAVE: expectativas trabalhistas - profissionalização - estudantes universitários - trabalho - educação.

\section{Cómo citar el artículo:}


Castañón Octavio, N., González Reyes, G., y Lupo Carbone, V. Estudio comparativo sobre las expectativas laborales de estudiantes de la carrera de educación inicial y los estudiantes de profesionalización en servicio en la Universidad Metropolitana

Castañón Octavio, N., González Reyes, G., y Lupo Carbone, V. (2020). Estudio comparativo sobre las expectativas laborales de estudiantes de la carrera de educación inicial y los estudiantes de profesionalización en servicio en la Universidad Metropolitana. [Comparative study about labor expectations of students of the initial education career and professionalization at the Metropolitan University] Revista de Comunicación de la SEECI, 53, 53-67. doi: https://doi.org/10.15198/seeci.2020.53.53-67

Recuperado de http://www.seeci.net/revista/index.php/seeci/article/view/653

\section{INTRODUCCIÓN}

En virtud de los resultados del trabajo investigativo de Ocanto y Tirado (2018) y de Del Valle y García García (2020) se quiere conocer en el presente trabajo de investigación, cuáles son, o si existen, las diferencias entre las expectativas laborales de los estudiantes de profesionalización en servicio con los estudiantes de educación inicial.

El programa de profesionalización en servicio de la UNIMET tiene como propósito, así como lo indica el sitio web de la UNIMET (s/f)

Propósito educativo:

Este estudio comparativo tiene como procedente el trabajo de investigación previo de Ocanto y Tirado (2018) en donde se detectaron las expectativas laborales de los estudiantes de educación inicial y se tiene que: "Como respuesta generalizada, el deseo de los estudiantes de convertirse en agente de cambio y desempeñar su rol docente $(90,24 \%)$ a pesar de considerar que la carrera no es valorada por la sociedad venezolana"(p.101)Aunado a lo anterior, "se evidenció que el 47,62\% puntualizó como oportunidad trabajo deseado la gerencia educativa" (p.102).

También se tuvo que la competencia que recibió mayor puntaje fue la que responde al docente como agente de cambio social, en tres dimensiones: educativa, legal y cultural (63 puntos en total). Los estudiantes no otorgaron la mayor valoración a la competencia que por excelencia sustenta el rol docente, la planificación de la educación (46) y la evaluación (48). (Ocanto y Tirado, (2018) concluyen que: "el determinar las expectativas laborales de los estudiantes de la carrera de Educación Inicial en la UNIMET, se le otorga una valoración fundamental para contribuir al proceso de mejoramiento continuo de la calidad académica, puesto que, desde el conocimiento de las realidades, intereses, necesidades, y evidentemente, las tres expectativas que se estudiaron en la presente investigación, es que realmente se pueden elaborar planes de acción funcionales, en pro de la excelencia académica y laboral de la población unimetana, y el dinamismo del mercado laboral" (p.105.)

Se consideraron relevantes los resultados al verificar las expectativas materiales de estos ya que el mercado laboral es dinámico, cambiante y demandante, por lo 
Castañón Octavio, N., González Reyes, G., y Lupo Carbone, V. Estudio comparativo sobre las expectativas laborales de estudiantes de la carrera de educación inicial y los estudiantes de profesionalización en servicio en la Universidad Metropolitana

que, es necesario que los docentes cuenten con formación actualizada y permanente.

\section{METODOLOGÍA}

\subsection{Diseño de la investigación}

El diseño de una investigación es "una estrategia general que tiene como finalidad imponer los controles necesarios para lograr un máximo de validez interna y externa(...)" (p.1) este se define con base en el procedimiento (Castañón, 2019).

Esta investigación posee un diseño no experimental, ya que se lleva a cabo sin manipular variables de manera deliberada.

Se estudian las expectativas laborales de los estudiantes de Profesionalización en Servicio de la UNIMET y después de haber analizado estos datos, se realiza una comparación con las expectativas laborales de los 69 estudiantes de educación inicial de la misma institución, estos datos fueron previamente recopilados en el trabajo de investigación de Ocanto y Tirado (2018).

En este diseño no experimental se observarán y se analizarán situaciones que ya existen, estas no son provocadas de manera intencional por el investigador y tampoco son manipulables.

Aunado a esto, este estudio es de tipo transversal ya que los datos son recolectados en un único momento (Castañón, 2019).

\subsection{Tipo de investigación}

Esta corresponde a un tipo de investigación comparativa y descriptiva, es comparativa debido a que se van a comparar las expectativas laborales de dos grupos, el de estudiantes de la carrera de educación inicial en la UNIMET y los estudiantes que cursan el programa de profesionalización de la misma institución.

Castañón (2019) afirma que un tipo de investigación comparativa está orientada a destacar la forma diferencial en la cual un fenómeno se manifiesta en grupos o contextos determinados y que, aunado a esto, permite detectar variables que luego se pueden utilizar para investigaciones confirmatorias.

A la vez es un tipo de investigación descriptiva debido a que esta busca especificar las propiedades importantes, referente a las expectativas laborales de los estudiantes de profesionalización en servicio de la UNIMET.

Según Castañón (2019) una investigación de tipo descriptiva trata de medir de forma independiente los conceptos o variables a que se refieren sin establecer relaciones causa- efecto, aunado a esto, requieren de conocimiento previo teórico e investigativo para poder construir instrumentos de medida válidos y confiables. 
Castañón Octavio, N., González Reyes, G., y Lupo Carbone, V. Estudio comparativo sobre las expectativas laborales de estudiantes de la carrera de educación inicial y los estudiantes de profesionalización en servicio en la Universidad Metropolitana

\subsection{Variables, dimensiones e indicadores}

Las siguientes variables presentadas, se centran en los estudiantes que actualmente cursan el programa de Profesionalización en Servicio de la UNIMET, y sus expectativas laborales buscando la relación entre ambas sin que las investigadoras interfieran en ellas.

Las variables, dimensiones e indicadores de esta investigación se presentan en el cuadro a continuación:

Tabla 1. Variables, dimensiones e indicadores.

\begin{tabular}{|c|c|c|}
\hline Variables & Dimensiones & Indicadores \\
\hline \multirow{3}{*}{$\begin{array}{l}\text { Sujetos universitarios del } \\
\text { programa de } \\
\text { Profesionalización en } \\
\text { Servicio de la } \\
\text { Universidad } \\
\text { Metropolitana }\end{array}$} & Perfil Personal & $\begin{array}{l}\text { Edad. } \\
\text { Género. } \\
\text { Nivel de Ingresos: métodos de pago } \\
\text { de matrícula, ocupación del } \\
\text { responsable económico, vivienda, } \\
\text { estratos sociales. } \\
\text { Intereses Personales: aspiraciones, } \\
\text { postgrados, máster. }\end{array}$ \\
\hline & Perfil Académico & $\begin{array}{l}\text { Empleo Actual. } \\
\text { Índice académico. } \\
\text { Capacidades requeridas. } \\
\text { Oportunidades de trabajo. } \\
\text { Vías de ingreso. }\end{array}$ \\
\hline & Perfil Laboral & $\begin{array}{l}\text { Experiencias laborales pasadas. } \\
\text { Empleo actual. } \\
\text { Competencias. }\end{array}$ \\
\hline \multirow{3}{*}{ Expectativas Laborales } & Materiales & $\begin{array}{l}\text { Salario deseado. } \\
\text { Puesto donde se desempeña. } \\
\text { Lugar donde trabaja: oficinas, } \\
\text { gerencia educativa, centros educativos } \\
\text { públicos o privados. }\end{array}$ \\
\hline & Sociales & $\begin{array}{l}\text { Notoriedad. } \\
\text { Puesto que desea desempeñar. } \\
\text { Lugar donde trabaja. }\end{array}$ \\
\hline & Realización Personal & $\begin{array}{l}\text { Inclinación humanitaria. } \\
\text { Puesto que desea desempeñar. } \\
\text { Lugar donde trabaja. }\end{array}$ \\
\hline
\end{tabular}


Castañón Octavio, N., González Reyes, G., y Lupo Carbone, V. Estudio comparativo sobre las expectativas laborales de estudiantes de la carrera de educación inicial y los estudiantes de profesionalización en servicio en la Universidad Metropolitana

\begin{tabular}{|l|l|} 
Condiciones Laborales & $\begin{array}{l}\text { Contrato: beneficios que le prestará al } \\
\text { trabajador, remuneración a recibir, } \\
\text { cargo a desempeñar. }\end{array}$ \\
\hline
\end{tabular}

Fuente: Elaboración propia.

\subsection{Población y Muestra}

Los estudiantes que se encuentren en el programa de profesionalización en servicio en la Universidad Metropolitana son aquellos que constituyen la población de este estudio, siendo un total de 68 estudiantes. La directora de la escuela de educación a la fecha de 14 de mayo de 2019 fue quien suministró estos datos confirmados para la investigación. La población es la totalidad del fenómeno a estudiar, donde las unidades de población poseen una característica en común, la que se estudia y da origen a los datos de la investigación (Castañón, 2019).

Castañón (2019) señala que la muestra es una selección de un grupo más pequeño de sujetos que representa a la población. En esta oportunidad, se utilizará el muestreo intencional por conveniencia, el cual, para Castañón (2019a) implica una población cautiva de la cual se seleccionan sujetos bajo un criterio que establece el investigador.

\begin{tabular}{|c|c|c|c|c|}
\hline $\begin{array}{c}\text { Tipo de } \\
\text { muestreo }\end{array}$ & $\begin{array}{c}\text { Estrategia de } \\
\text { muestreo }\end{array}$ & Debe usarse & Ventajas & Desventajas \\
\hline $\begin{array}{c}\text { No } \\
\text { probabilístico }\end{array}$ & $\begin{array}{c}\text { Muestreo de } \\
\text { conveniencia }\end{array}$ & $\begin{array}{c}\text { Cuando la } \\
\text { muestra es } \\
\text { cautiva }\end{array}$ & $\begin{array}{c}\text { Cómodo y } \\
\text { económico }\end{array}$ & $\begin{array}{c}\text { La representatividad } \\
\text { es dudosa }\end{array}$ \\
\hline
\end{tabular}

El criterio de muestra para este trabajo de investigación es el de los estudiantes activos que actualmente cursen el programa de profesionalización en servicio en la Universidad Metropolitana, asimismo, sus años de servicio y titularidad en cargos desempeñados.

\subsection{Instrumento de recolección y análisis de los datos}

El instrumento de recolección constituye la principal fuente de obtención de los datos, los cuales se fundamentan en las variables estudiadas, este arroja resultados de carácter cuantitativo, pero a su vez, la medición de resultados cualitativos. (Ocanto y Tirado, 2018)

En esta investigación se realizará una encuesta como el instrumento de recolección de datos, la encuesta se puede definir como "una investigación realizada sobre una muestra de sujetos representativa de un colectivo más amplio, utilizando 
Castañón Octavio, N., González Reyes, G., y Lupo Carbone, V. Estudio comparativo sobre las expectativas laborales de estudiantes de la carrera de educación inicial y los estudiantes de profesionalización en servicio en la Universidad Metropolitana

procedimientos estandarizados de interrogación con el fin de obtener mediciones cuantitativas de una gran variedad de características objetivas y subjetivas de la población" (Torres, y col. (2014))

Para (Torres et al, 2014) entre las características fundamentales de una encuesta se destacan:

1. La encuesta es una observación no directa de los hechos por medio de lo que manifiestan los interesados.

2. Es un método preparado para la investigación.

3. Permite una aplicación masiva que mediante un sistema de muestreo pueda extenderse a una nación entera.

4. Hace posible que la investigación social llegue a los aspectos subjetivos de los miembros de la sociedad.

La encuesta utilizada en esta oportunidad fue adaptada, del instrumento previamente elaborado para la investigación realizada por el equipo de investigación de la profesora Natalia Castañón.

\section{OBJETIVOS}

Debido a que este trabajo es un estudio comparativo entre las expectativas laborales de los estudiantes de educación inicial y los estudiantes de profesionalización en servicio, es importante destacar que 7 el propósito del programa de profesionalización en servicio que ofrece la Universidad Metropolitana es el de "Proporcionar a los docentes en ejercicio la oportunidad para desarrollar las competencias necesarias para el logro de altos niveles de eficacia y eficiencia en su rol como educador; permitiéndole asumir un papel protagónico como agente de cambio pedagógico y social en el contexto educativo venezolano". (UNIMET (s/f) propósito del programa de profesionalización en servicio)

Finalmente, respondiendo a los resultados de las investigaciones pasadas y con la intención de seguir esta línea de investigación, se desea responder la siguiente interrogante: ¿Cuáles son los factores que influyen en las expectativas laborales de los estudiantes de la modalidad de profesionalización en servicio? ¿Cuál es la diferencia o semejanza entre los estudiantes de pregrado regulares de educación inicial y los estudiantes de la modalidad de profesionalización en servicio en cuanto a sus expectativas laborales?

\section{RESULTADOS}

Posteriormente de haber culminado los análisis de las figuras y las tablas, se considera relevante resaltar las relaciones más importantes que se desprenden de los resultados provenientes del instrumento de recolección de datos con su respectiva comparación a los resultados obtenidos por Ocanto y Tirado en 2018.

- Se tiene que el 59,5\% (25) de los estudiantes de profesionalización en servicio de la UNIMET ingresaron a la universidad por el proceso de plan de becas que ofrece la misma. Por otro lado, tal como lo establecieron Ocanto y Tirado en el 
Castañón Octavio, N., González Reyes, G., y Lupo Carbone, V. Estudio comparativo sobre las expectativas laborales de estudiantes de la carrera de educación inicial y los estudiantes de profesionalización en servicio en la Universidad Metropolitana

2018, se tiene que el $80,95 \%$ (17) de los estudiantes de educación inicial utilizó como vía de ingreso el examen de ubicación, diagnóstico y evaluación integral.

- Se tiene que el $83,3 \%$ (35) de los estudiantes de profesionalización afirman considerar que estudiar en este programa va efectivamente a ayudarlos a conseguir su trabajo anhelado, demostrando la satisfacción que posee este porcentaje de estudiantes de sus expectativas laborales y metas personales. Aunado a esto se tiene que el $64,3 \%$ (27) de los estudiantes de profesionalización consideran que necesitan de una titulación universitaria en docencia para mejorar su desempeño profesional, lo cual llama la atención debido a que el $71,4 \%$ (30) de los estudiantes consideran que la carrera de educación no es valorada por la sociedad venezolana. Complementando la idea anterior, tal como lo establecieron Ocanto y Tirado en 2018, el 95,24\% de las estudiantes de educación inicial también consideraban que la carrera no es valorada por la sociedad venezolana y sin embargo, el $100 \%$ de las estudiantes consideraban que estudiar la carrera de educación podía convertirlas en un agente de cambio social. Por lo que se refleja una semejanza en ambos grupos, se puede indicar que a pesar de la poca valoración que posee la carrera en la sociedad venezolana, ambas muestras siguen formándose como educadores. Sin embargo, se establece una diferencia, en el grupo de las estudiantes de educación inicial el 100\% (21) de la población consideraba que seguir sus estudios de la carrera las ayudará a elegir su trabajo anhelado, sin embargo, el porcentaje de estudiantes de profesionalización que considera que puede conseguir su trabajo anhelado estudiando la carrera es de, como fue mencionado anteriormente de, $83,3 \%$.

- Como beneficios adicionales a la remuneración salarial, se tiene que el $85,7 \%$ (36) estudiantes de profesionalización desean contar con incentivos de rendimiento y desempeño, asimismo el $76,2 \%$ (32) estudiantes de profesionalización desean contar con cursos de actualización, el 73,8\% (31) estudiantes de profesionalización desean contar con beneficios generales tal como lo es el transporte y siguiendo el mismo orden de ideas el $57,1 \%$ (24) estudiantes desean contar con bonos de alimentación. En semejanza con, tal como lo plasmaron Ocanto y Tirado en 2018 , se destaca la coherencia que existe con el $80,95 \%$ (17) estudiantes de educación inicial que quisieran contar con cursos de actualización, bonos de alimentación y beneficios generales como lo es el transporte.

- Se puede observar que los estudiantes ingresados a la carrera en el programa de profesionalización en servicio entre los años 2014-2016 suma un total de (26) estudiantes lo que representa un $61,9 \%$ de la muestra. Mientras que, en el trabajo de investigación de Ocanto y Tirado en 2018, las estudiantes ingresadas a la carrera entre los años 2014-2016 sumaba un total de (13) estudiantes que corresponde al $61,90 \%$ de la carrera.

- Cabe destacar que los estudiantes pertenecientes al programa de profesionalización en servicio desean desempeñarse mayoritariamente en la docencia y en capacitación, investigación, coordinación y gerencia de centros educativos y programas sociales o centros culturales y recreativos. Mientras que, tal como lo señalaron Ocanto y Tirado en su trabajo de investigación en 
Castañón Octavio, N., González Reyes, G., y Lupo Carbone, V. Estudio comparativo sobre las expectativas laborales de estudiantes de la carrera de educación inicial y los estudiantes de profesionalización en servicio en la Universidad Metropolitana

el 2018, las estudiantes de educación inicial aspiran a cargos directivos y de mayor jerarquía.

- Se tiene un 33,3\% (14) estudiantes de profesionalización que desean ejercer la docencia en otro país diferente a Venezuela, lo que refleja un contraste a las estudiantes de educación inicial, según lo establecido por las autoras Ocanto y Tirado en 2018 , debido a que el $57,14 \%$ (12) estudiantes también desean ejercer la profesión de educación, fuera de Venezuela.

- Al momento de solventar el pago de su matrícula universitaria, se tiene que el $66,7 \%$ (28) de los estudiantes de profesionalización utilizan el programa de becas estudiantiles, contrastando con lo establecido por Ocanto y Tirado en 2018 , ya que el $90,48 \%$ establece que solventan el pago de su matrícula mediante sus familiares o por cuenta propia.

- Se tiene que el $88,1 \%$ (37) de los encuestados pertenecientes al grupo de estudiantes de profesionalización preferiría trabajar en un centro educativo privado, aunado a esto, según lo establecido por Laura Ocanto y Valerie Tirado en 2018, el 95,24\% (20) de las estudiantes de educación inicial también preferirían trabajar en un centro educativo privado.

- Se obtuvo un 51,2\% (22) de los estudiantes de Profesionalización en Servicio que desean ejercer en la docencia al poseer el título de licenciado en educación, asimismo se observa similitud con las estudiantes de Educación Inicial de las que el 95,24\% desean ejercer en la docencia al poseer el título de licenciado en educación.

- Aunado a esto, se tiene que $53,5 \%$ (23) de los estudiantes de profesionalización desean desempeñarse en capacitación, investigación, coordinación y gerencia de centros educativos y programas sociales, centros culturales y recreativos, al obtener el título de licenciado en educación. En coherencia con lo establecido por Ocanto y Tirado en 2018 donde se observa que el $52,38 \%$ (11) de las estudiantes de Educación Inicial igualmente desean desempeñarse en capacitación, investigación, coordinación y gerencia de centros educativos y programas sociales, centros culturales y recreativos, al obtener el título de licenciado en educación.

- Al puntualizar su oportunidad de trabajo deseada, se obtuvo que el $11,9 \%$ (5) de los estudiantes de Profesionalización desean desempeñarse como Gerentes educativos, en contraste con las estudiantes de Educación Inicial representando un $47,62 \%$ que al señalar su oportunidad de trabajo deseada, desean desempeñarse también como Gerentes educativos.

- Aunado a lo anterior, el 83,7\% (36) de los estudiantes de Profesionalización en Servicio afirman que estudiar en este programa los ayudará a encontrar su trabajo anhelado. Por otra parte, según lo previamente investigado por Ocanto y Tirado, el $100 \%$ de las estudiantes de Educación Inicial afirman que estudiar educación inicial en la Universidad Metropolitana las ayudará a encontrar su trabajo anhelado.

- Según las respuestas obtenidas, se puede afirmar que el 69,8\% (30) de los estudiantes de Profesionalización en Servicio consideran que la carrera de educación no es valorada por la sociedad venezolana, en concordancia con lo establecido en 2018 por Ocanto y Tirado, 95,24\% (20) de las estudiantes de 
Castañón Octavio, N., González Reyes, G., y Lupo Carbone, V. Estudio comparativo sobre las expectativas laborales de estudiantes de la carrera de educación inicial y los estudiantes de profesionalización en servicio en la Universidad Metropolitana

Educación Inicial consideran, de igual manera, que la carrera de educación no es valorada por la sociedad venezolana.

- Para mejorar su desempeño como docente, el 65,1\% (28) de los estudiantes de Profesionalización en Servicio consideran necesario una titulación en educación. En la misma línea de ideas, para desempeñarse como docente, 80,95\% (17) de las estudiantes de Educación Inicial consideran necesaria una titulación en educación.

- Asimismo, el 88,4\% (38) de los estudiantes de Profesionalización en Servicio y el $95,24 \%$ (20) de las estudiantes de Educación Inicial desean desempeñarse como profesionales de la educación en un centro educativo privado.

- En el mismo orden de ideas, el $86 \%$ (37) de los estudiantes de Profesionalización en Servicio y el $95,48 \%$ (19) de las estudiantes de Educación Inicial, según lo establecido por Ocanto y Tirado en el 2018, desean obtener un contrato a tiempo determinado a la hora de laborar.

- Aunado a la remuneración salarial, al $83,7 \%$ (36) de los estudiantes de Profesionalización y al $71,43 \%$ (15) de las estudiantes de Educación Inicial les gustaría recibir beneficios de tipo incentivos de rendimiento y desempeño a la hora de laborar.

- Una vez siendo Licenciado en Educación, el 67,4\% (29) de los estudiantes de profesionalización y, según Ocanto y Tirado en 2018, 61,90\% (13) de las estudiantes de Educación Inicial desean ejercer en un país distinto a Venezuela. Siendo España la opción más tentativa por ambos grupos, con un $28,57 \%$ en las estudiantes de Educación Inicial y un $21,4 \%$ en los estudiantes de Profesionalización en Servicio.

\section{DISCUSIÓN}

En la presente investigación se puede concluir que existen múltiples expectativas sociales, personales y materiales pertenecientes a los estudiantes de profesionalización en servicio que se asemejan y se diferencian en muchas expectativas a los estudiantes de Educación Inicial. Destacando todas estas expectativas laborales con la principal diferencia entre ambos grupos, la cual es que los estudiantes de profesionalización en servicio, en su mayoría, poseen experiencia laboral y se están desempeñando en la actualidad como docentes, mientras que, las estudiantes de Educación Inicial entran en la carrera con poca o ninguna experiencia laboral.

Tomando en consideración nuestro primer objetivo específico: Indagar acerca de las expectativas laborales de los estudiantes del programa de Profesionalización en Servicio. Se obtuvo que:

En los resultados obtenidos en esta investigación se refleja que existe una fuerte tendencia, exactamente el 83,3\%, de los estudiantes de Profesionalización al considerar que estudiar en este programa va a ayudarlos a conseguir su trabajo deseado. A pesar de que la gran mayoría de los estudiantes consideran que la carrera de Educación no es valorada por la sociedad venezolana, más de la mitad de 
Castañón Octavio, N., González Reyes, G., y Lupo Carbone, V. Estudio comparativo sobre las expectativas laborales de estudiantes de la carrera de educación inicial y los estudiantes de profesionalización en servicio en la Universidad Metropolitana

la muestra, 64,3\%, sigue afirmando la necesidad que se tiene que titularse como educador para mejorar su desempeño profesional, lo que demuestra que las expectativas sociales son minimizadas a la hora de compararse con sus expectativas personales y se estima un nivel alto en la valorización del empleo de parte de la mayoría de los estudiantes de Profesionalización. Aunado a esto, se obtuvo que la mayoría de los estudiantes de profesionalización preferiría trabajar en un centro educativo privado y que su trabajo sea retribuido en dólares.

Por otra parte, realizar un sondeo acerca del nivel económico que consideran los estudiantes de Profesionalización que pertenece su vivienda asimismo como el responsable de solventar su matrícula universitaria fue relevante para la investigación debido a que mientras los estudiantes acepten su nivel de vida, más ajustadas serán sus expectativas laborales. Asimismo, el $45,2 \%$ se consideró su vivienda en el nivel medio alto y el 40,5\% consideró su vivienda en el nivel medio. Aunado a lo anterior, el $66,7 \%$, que corresponde a más de la mitad de la muestra solventa su matrícula mediante una beca universitaria. Lo cual refleja una diferencia marcada con las estudiantes de educación inicial debido a que estas, solventan el pago de su matrícula mediante familiares en su mayoría, exactamente el 90,48\%. Sin embargo, las estudiantes de educación inicial, así como lo investigaron las autoras Ocanto y Tirado, ubican su nivel de vivienda en el Alto (19,05\%), Medio alto $(52,38 \%)$ y medio $(28,57 \%)$.

Al mismo tiempo, se evidenció que el 33,3\%, la mayoría de las respuestas puntualizó como trabajo deseado la docencia. Para las autoras, significa que sus expectativas laborales no son exactamente altas, es decir, poseen expectativas ajustadas a su profesión, sin embargo, no de alta gama. Lo que conduce a otra diferencia establecida con las estudiantes de Educación Inicial, según Ocanto y Tirado en 2018 , estas puntualizan con un $47,62 \%$ como trabajo deseado la gerencia educativa, por cuanto se proyectan en el escalón más alto de la jerarquía educativa.

Nuevamente, se refleja en la investigación que las expectativas materiales y sociales de los estudiantes de Profesionalización son altas debido a que el $40,5 \%$ consideró como una retribución salarial debida entre $700 \$-1000 \$$ al mes en Venezuela y el 33,3\% consideró como una retribución salarial debida entre 500 $700 \$$ al mes en en mismo país, lo que indica que el $73,8 \%$ de los estudiantes de Profesionalización en servicio consideran que el pago debe estar entre los $500 \$$ a $1000 \$$. Por lo cual, se tomaron precauciones de que los encuestados desearían tener ciertas condiciones laborales previamente determinadas, siendo seleccionado el $85,7 \%$ los contratos a tiempo determinado, donde se aspiran beneficios anteriormente mencionados.

En cuanto al segundo objetivo específico: Determinar el perfil de ingreso de los estudiantes del Programa de Profesionalización en Servicio.

Se tiene que el 60,5\% (26) de los estudiantes de Profesionalización en Servicio ingresaron a la Universidad Metropolitana por el Proceso de Plan de Becas que ofrece la misma. Mientras que, según lo anteriormente investigado por Ocanto y Tirado en 
Castañón Octavio, N., González Reyes, G., y Lupo Carbone, V. Estudio comparativo sobre las expectativas laborales de estudiantes de la carrera de educación inicial y los estudiantes de profesionalización en servicio en la Universidad Metropolitana

2018, el 80,95\% ingresaron a la carrera mediante el examen diagnóstico, ubicación y evaluación integral, de las cuales el $46,82 \%$ ingresaron en las listas 2 , con un $23,41 \%$ y 4 , con un $23,41 \%$ respectivamente.

Correspondiente al tercer objetivo específico, responde a: Constatar las expectativas laborales de la carrera de Educación Inicial y del programa de Profesionalización en Servicio. Se obtuvo que existe similitud entre los estudiantes de Profesionalización en Servicio y los estudiantes de Educación Inicial ya que se demostró que la mayoría de ambos grupos, como lo demuestran las estadísticas anteriormente, son coherentes con muchas de las expectativas materiales, sociales y personales diferenciándose en una característica, ya que al puntualizar su oportunidad de trabajo deseada, se obtuvo que los estudiantes de Educación Inicial se inclinaron más a su oportunidad de trabajo deseada a la Gerencia Educativa, mientras que los Estudiantes de Profesionalización se inclinaron por la docencia.

\section{CONCLUSIONES}

Tomando en cuenta el objetivo general: comparar las expectativas laborales de los estudiantes de la carrera de educación inicial con las de los estudiantes del programa de profesionalización en servicio de la Universidad Metropolitana: se puede concluir que se tienen que determinar las expectativas laborales de los estudiantes y compararlas, debido a que contribuye al mejoramiento continuo de la calidad académica educativa ya que al conocer las distintas realidades de los estudiantes se entienden sus necesidades y sus intereses. Aunado a que, al conocer sus expectativas, se pueden elaborar planes de acción para elevarlas, hacerlas posibles 0 tangibles, siempre en pro de la excelencia académica que se desea logren los estudiantes.

\section{REFERENCIAS}

Balestrini, M. (2002). Cómo se elabora el proyecto de investigación. Caracas: BL Consultores asociados.

Bonillo, D. y Nieto, F. (2003). La Satisfacción Laboral como Elemento Motivador del Empleado. Trabajo, 11, 189-200. Recuperado de http://rabida.uhu.es/dspace/bitstream/handle/10272/2421/b13772089.pdf?sequen $\underline{\mathrm{ce}=1}$

Corica, A. (2012). Las expectativas sobre el futuro educativo y laboral de jóvenes de la escuela secundaria: entre lo posible y lo deseable. Última década, 36, 71-95. Recuperado de: https://scielo.conicyt.cl/pdf/udecada/v20n36/art04.pdf

Facultad de Ciencias Jurídicas y Políticas [FCJP] (2013). Anteproyecto de Ley de Educación Universitaria (FCJP). Venezuela: Universidad del Zulia. Recuperado de: http://www.luz.edu.ve/index.php?option=com content\&view=category\&id=103\&I temid $=508$ 
Castañón Octavio, N., González Reyes, G., y Lupo Carbone, V. Estudio comparativo sobre las expectativas laborales de estudiantes de la carrera de educación inicial y los estudiantes de profesionalización en servicio en la Universidad Metropolitana

Farné, S. (2011). Observatorios del mercado de trabajo: ¿Qué son y cómo funcionan?. Economía Institucional, 13(24), 429-445. Recuperado de: https://dialnet.unirioja.es/descarga/articulo/3668789.pdf

Fidias, A. (2012). El Proyecto de Investigación: Introducción a la metodología científica. Caracas: Episteme.

Guevara, M. (2014). Mi primera búsqueda de empleo. El proceso a conocer. Observatorio Laboral Revista Venezolana, $\lambda_{14}$ ), 35-49. Recuperado de: http://servicio.bc.uc.edu.ve/faces/revista/lainet/lainetv7n14/art02.pdf

Hernández, R., Fernández, C. y Baptista, P. (2010). Metodología de la investigación. México D.F.: McGraw-Hill.

Juárez, J. y García, E. (2015). Contexto educativo y formación docente en Venezuela. Escuela de Educación Universidad Católica Andrés Bello. Foro Cerpe-Serie Educalidad, 8.

Pérez, C. (2015). Inserción Laboral de Universitarios desde la Perspectiva Psicosocial. Universidad de Extremadura.

Rodríguez, N. (2004). Retos de la formación de docentes en Venezuela. Revista Pedagógica, 25(73). Recuperado de: www.scielo.org.ve/scielo.php?script=sci arttext\&pid=S0798-97922004000200002

Sieglin, V. y Zúñiga, M. (2010). Brain Drain en México: estudio sobre expectativas de trabajo y disposición hacia la migración laboral en estudiantes de Ingeniería y Ciencias Naturales. Perfiles educativos, 32(128), 55-79. Recuperado de: www.scielo.org.mx/pdf/peredu/v32n128/v32n128a4.pdf

Steinmann, A., Bosch, B. y Aiassa, D. (2013). Motivación y Expectativas de los Estudiantes por Aprender Ciencias en la Universidad. Recuperado de: http://www.scielo.org.mx/pdf/rmie/v18n57/v18n57a12.pdf

Suárez, B. (2016). Éxodo intelectual en el ámbito venezolano: una mirada desde la percepción de los estudiantes universitarios. Venezuela en la mira de las ciencias económicas y sociales. Encuentro de saberes, 6, 1451-1462. Recuperado de: http://mriuc.bc.uc.edu.ve/bitstream/handle/123456789/4300/tomo6.pdf

Suriá, R., Rosser, A. y Villegas, E. (2013b). Diferencias en actitudes y expectativas de futuro laboral antes y después de haber iniciado estudios universitarios. XI Jornadas de redes de investigación en docencia universitaria. Recuperado de: https://web.ua.es/es/ice/jornadas-redes/documentos/2013-comunicacionesorales/334594.pdf

UNIMET. (s/f). Plan de Estudio Licenciatura en Educación Mención Educación Inicial. Recuperado de: http://www.unimet.edu.ve/educacion/plan-de-estudiolicenciatura-en-educacion-mencion-educacion-inicial/ 
Castañón Octavio, N., González Reyes, G., y Lupo Carbone, V. Estudio comparativo sobre las expectativas laborales de estudiantes de la carrera de educación inicial y los estudiantes de profesionalización en servicio en la Universidad Metropolitana

Universia. (2015). La Importancia de Conocer tu Motivación Laboral para tener Hábitos Productivos. Recuperado de: https://noticias.universia.com.ar/consejosprofesionales/noticia/2015/07/15/1128357/importancia-conocer-motivacionlaboral-tener-habitos-productivos.html

Valle, A., Regueiro, B., Rodríguez, S., Piñeiro, I., Freire, C., Ferradas, S. y Suárez, N. (2015). Perfiles motivacionales como combinación de expectativas de autoeficacia y metas académicas en estudiantes universitarios. European Journal of Education and Psychology, 8(1), 1-8. doi: 10.1016/j.ejeps.2015.10.001

Vázquez, A. y Manassero, M. (2009). Expectativas sobre un trabajo futuro y vocaciones científicas en estudiantes de educación secundaria. Revista Electrónica de Investigación Educativa, 11(1). Recuperado a partir de https://redie.uabc.mx/index.php/redie/article/view/218

Vázquez, A. y Manassero, M. (2008). Validación de un cuestionario de expectativas laborales con estudiantes de secundaria. Psicothema, 20(4), 659-664. Recuperado de http://www.psicothema.com/psicothema.asp?id=3537

Velásquez, E., Loreto M. y Cumsille, P. (2004). Expectativas de autoeficacia y actitud prosocial asociadas a participación ciudadana en jóvenes. Psykhe, 13(2), 85-98. doi: $10.4067 /$ S0718-22282004000200007

Weller, J. (2007). La Inserción Laboral de los Jóvenes: Características, Tensiones y Desafíos. Recuperado de: https://repositorio.cepal.org/bitstream/handle/11362/11192/092061082 es.pdf?se quence $=1$ \&isAllowed $=y$

\section{AUTORAS}

\section{Natalia Castañón Octavio}

Profesora e investigadora a tiempo completo en la Universidad Metropolitana (1993). Fundadora de tuterapiaenlinea.com (2014-). Licenciada en Educación (Universidad Metropolitana, 1991). Maestría en Psicología Escolar (Universidad Complutense de Madrid 1993). Doctorada en Filosofía y Ciencias de la Educación (Universidad Complutense de Madrid, 1999). Ha realizado carrera docente, gerencial e investigación: jefa del departamento de Didáctica (2002-), jefa del departamento de Humanidades (2014-), gerente general del centro de aprendizaje en línea (2014) y profesora investigadora. Ha sido tutora de treinta y siete tesis de pregrado, maestría y doctorado. Publicaciones en veinte y dos revistas o libros arbitrados a nivel nacional e internacional. Ha participado en cuarenta congresos nacionales e internacionales con ponencias arbitradas.

ncastanon@unimet.edu.ve

Orcid ID: https://orcid.org/0000-0002-7532-6924 
Castañón Octavio, N., González Reyes, G., y Lupo Carbone, V. Estudio comparativo sobre las expectativas laborales de estudiantes de la carrera de educación inicial y los estudiantes de profesionalización en servicio en la Universidad Metropolitana

\section{Giovanna González Reyes}

Fue docente de inglés como lengua extranjera en el Club Hebraica Caracas (2019). Licenciada en Educación de la Universidad Metropolitana en el 2020 (CaracasVenezuela). Posee un diplomado en Digital Marketing realizado en la Universidad Metropolitana y un diplomado de Educación multidisciplinaria y terapéutica en Diabetes realizado en la Universidad Central de Venezuela. Aspirante la maestría de Psicopedagogía en la Universidad de Valencia, España.

giovannagonzalez@correo.unimet.edu.ve

\section{Valeria Lupo Carbone}

Fue auxiliar de docente en el Instituto Educacional Henry Clay (2015-2016). Licenciada en Educación de la Universidad Metropolitana en el 2020 (Caracas Venezuela). También se desempeñó como ayudante de docente en el maternal CEIWEI (2014-2015). Aspirante a posgrado en Psicología en la Universidad de Tampa, Estados Unidos. Y aspirante a maestría en Psicología Positiva en la Universidad de Palermo, Argentina.

valerialupo@correo.unimet.edu.ve 